\title{
Search for Chargino and Neutralino Production Using the OPAL Detector at $\sqrt{s}=130-136 \mathrm{GeV}$ at LEP The OPAL Collaboration
}

\begin{abstract}
A search for charginos and neutralinos, predicted by supersymmetric theories, has been performed using a data sample of $2.6 \mathrm{pb}^{-1}$ at a centre-of-mass energy of $\sqrt{s}=130 \mathrm{GeV}$ and $2.6 \mathrm{pb}^{-1}$ at $136 \mathrm{GeV}$ collected with the OPAL detector at LEP during November 1995 . No candidate events were observed. The $95 \%$ C.L. lower limit on the lightest chargino mass in the Minimal Supersymmetric Standard Model is $65.4 \mathrm{GeV}$ if the universal scalar mass $m_{0}$ is greater than $1 \mathrm{TeV}$, and $58.7 \mathrm{GeV}$ for the smallest $m_{0}$ compatible with slepton and sneutrino mass limits obtained at centre-of-mass energies near the $\mathrm{Z}$ peak. These limits were obtained under the conditions that the lightest chargino is heavier than the lightest neutralino by more than $10 \mathrm{GeV}$ and $\tan \beta$ is larger than 1.5 . The results of a model independent search for charginos and neutralinos are also given.
\end{abstract}

Submitted to Physics Letters 
G. Alexander ${ }^{23}$, J. Allison ${ }^{16}$, N. Altekamp ${ }^{5}$, K. Ametewee ${ }^{25}$, K.J. Anderson ${ }^{9}$, S. Anderson ${ }^{12}$, S. Arcelli ${ }^{2}$, S. Asai ${ }^{24}$, D. Axen ${ }^{29}$, G. Azuelos ${ }^{18, a}$, A.H. Ball ${ }^{17}$, E. Barberio ${ }^{26}$, R.J. Barlow ${ }^{16}$, R. Bartoldus ${ }^{3}$, J.R. Batley ${ }^{5}$, G. Beaudoin ${ }^{18}$, J. Bechtluft ${ }^{14}$, C. Beeston ${ }^{16}$, T. Behnke ${ }^{8}$, A.N.Bell ${ }^{1}$, K.W.Bell ${ }^{20}$, G. Bella ${ }^{23}$, S. Bentvelsen ${ }^{8}$, P. Berlich ${ }^{10}$, S. Bethke ${ }^{14}$, O. Biebel ${ }^{14}$, V. Blobel ${ }^{8}$, I.J. Bloodworth ${ }^{1}$, J.E. Bloomer ${ }^{1}$, P. Bock ${ }^{11}$, H.M. Bosch ${ }^{11}$, M. Boutemeur ${ }^{18}$, B.T. Bouwens ${ }^{12}$, S. Braibant ${ }^{12}$, R.M. Brown ${ }^{20}$, H.J. Burckhart ${ }^{8}$, C. Burgard ${ }^{27}$, R. Bürgin ${ }^{10}$, P. Capiluppi ${ }^{2}$, R.K. Carnegie ${ }^{6}$, A.A. Carter $^{13}$, J.R. Carter ${ }^{5}$, C.Y. Chang ${ }^{17}$, C. Charlesworth ${ }^{6}$, D.G. Charlton ${ }^{1, b}$, D. Chrisman ${ }^{4}$, S.L. Chu ${ }^{4}$, P.E.L. Clarke ${ }^{15}$, I. Cohen ${ }^{23}$, J.E. Conboy ${ }^{15}$, O.C. Cooke $^{16}$, M. Cuffiani ${ }^{2}$, S. Dado ${ }^{22}$, C. Dallapiccola ${ }^{17}$, G.M. Dallavalle ${ }^{2}$, C. Darling ${ }^{31}$, S. De Jong $^{12}$, L.A. del Pozo ${ }^{8}$, M.S. Dixit ${ }^{7}$, E. do Couto e Silva ${ }^{12}$, E. Duchovni ${ }^{26}$, G. Duckeck ${ }^{8}$, I.P. Duerdoth ${ }^{16}$, J.E.G. Edwards ${ }^{16}$, P.G. Estabrooks ${ }^{6}$, H.G. Evans ${ }^{9}$, M. Evans ${ }^{13}$, F. Fabbri ${ }^{2}$, P. Fath ${ }^{11}$, F. Fiedler ${ }^{12}$, M. Fierro ${ }^{2}$, H.M. Fischer ${ }^{3}$, R. Folman ${ }^{26}$, D.G. Fong ${ }^{17}$, M. Foucher ${ }^{17}$, H. Fukui ${ }^{24}$, A.Fürtjes ${ }^{8}$, P. Gagnon ${ }^{7}$, A. Gaidot ${ }^{21}$, J.W. Gary ${ }^{4}$, J. Gascon ${ }^{18}$,

S.M. Gascon-Shotkin ${ }^{17}$, N.I. Geddes ${ }^{20}$, C. Geich-Gimbel ${ }^{3}$, S.W. Gensler ${ }^{9}$, F.X. Gentit ${ }^{21}$, T. Geralis ${ }^{20}$, G. Giacomelli ${ }^{2}$, P. Giacomelli ${ }^{4}$, R. Giacomelli ${ }^{2}$, V. Gibson ${ }^{5}$, W.R. Gibson ${ }^{13}$,

D.M. Gingrich ${ }^{30, a}$, J. Goldberg ${ }^{22}$, M.J. Goodrick ${ }^{5}$, W. Gorn ${ }^{4}$, C. Grandi ${ }^{2}$, E. Gross ${ }^{26}$, M. Gruwé ${ }^{8}$, C. Hajdu ${ }^{32}$, G.G.Hanson ${ }^{12}$, M. Hansroul ${ }^{8}$, M.Hapke ${ }^{13}$, C.K. Hargrove ${ }^{7}$, P.A.Hart ${ }^{9}$, C. Hartmann ${ }^{3}$, M. Hauschild ${ }^{8}$, C.M. Hawkes ${ }^{5}$, R. Hawkings ${ }^{8}$, R.J. Hemingway ${ }^{6}$, G. Herten ${ }^{10}$, R.D. Heuer ${ }^{8}$, M.D. Hildreth ${ }^{8}$, J.C.Hill ${ }^{5}$, S.J. Hillier ${ }^{1}$, T. Hilse ${ }^{10}$, P.R. Hobson ${ }^{25}$, R.J.Homer ${ }^{1}$,

A.K. Honma ${ }^{28, a}$, D. Horváth ${ }^{32, c}$, R. Howard ${ }^{29}$, R.E. Hughes-Jones ${ }^{16}$, D.E. Hutchcroft ${ }^{5}$, P. Igo-Kemenes ${ }^{11}$, D.C. Imrie ${ }^{25}$, M.R. Ingram ${ }^{16}$, A. Jawahery ${ }^{17}$, P.W. Jeffreys ${ }^{20}$, H. Jeremie ${ }^{18}$,

M. Jimack ${ }^{1}$, A. Joly ${ }^{18}$, G. Jones ${ }^{16}$, M. Jones ${ }^{6}$, R.W.L. Jones ${ }^{8}$, U. Jost ${ }^{11}$, P. Jovanovic ${ }^{1}$, J. Kanzaki ${ }^{24}$, D. Karlen ${ }^{6}$, T. Kawamoto ${ }^{24}$, R.K. Keeler ${ }^{28}$, R.G. Kellogg ${ }^{17}$, B.W. Kennedy ${ }^{20}$, J. King ${ }^{13}$, J. Kirk ${ }^{29}$, S. Kluth ${ }^{8}$, T. Kobayashi ${ }^{24}$, M. Kobel ${ }^{10}$, D.S. Koetke ${ }^{6}$, T.P. Kokott ${ }^{3}$, S. Komamiya ${ }^{24}$, R. Kowalewski ${ }^{8}$, T. Kress ${ }^{11}$, P. Krieger ${ }^{6}$, J. von Krogh ${ }^{11}$, P. Kyberd ${ }^{13}$, G.D. Lafferty ${ }^{16}$, H. Lafoux ${ }^{21}$, R. Lahmann ${ }^{17}$, W.P. Lai ${ }^{19}$, D. Lanske ${ }^{14}$, J. Lauber ${ }^{15}$, J.G. Layter ${ }^{4}$, A.M. Lee ${ }^{31}$, E. Lefebvre ${ }^{18}$, D. Lellouch ${ }^{26}$, J. Letts ${ }^{2}$, L. Levinson ${ }^{26}$, C. Lewis ${ }^{15}$, S.L. Lloyd ${ }^{13}$, F.K. Loebinger ${ }^{16}$, G.D. Long ${ }^{17}$, B. Lorazo ${ }^{18}$, M.J. Losty ${ }^{7}$, J. Ludwig ${ }^{10}$, A. Luig ${ }^{10}$, A. Malik ${ }^{21}$, M. Mannelli ${ }^{8}$, S. Marcellini ${ }^{2}$, C. Markus ${ }^{3}$, A.J. Martin ${ }^{13}$, J.P. Martin ${ }^{18}$, G. Martinez ${ }^{17}$, T. Mashimo ${ }^{24}$, W. Matthews ${ }^{25}$, P. Mättig ${ }^{3}$, W.J. McDonald ${ }^{30}$, J. McKenna ${ }^{29}$, E.A. Mckigney ${ }^{15}$, T.J.McMahon ${ }^{1}$, A.I. McNab ${ }^{13}$, F. Meijers ${ }^{8}$, S. Menke ${ }^{3}$, F.S. Merritt ${ }^{9}$, H. Mes ${ }^{7}$, J. Meyer $^{27}$, A. Michelini ${ }^{8}$, G. Mikenberg ${ }^{26}$, D.J.Miller ${ }^{15}$, R. Mir ${ }^{26}$, W. Mohr ${ }^{10}$, A.Montanari ${ }^{2}$, T. Mori ${ }^{24}$, M. Morii ${ }^{24}$, U. Müller ${ }^{3}$, B. Nellen ${ }^{3}$, B. Nijjhar ${ }^{16}$, R. Nisius ${ }^{8}$, S.W. O'Neale ${ }^{1}$, F.G. Oakham ${ }^{7}$, F. Odorici ${ }^{2}$, H.O. Ogren ${ }^{12}$, T. Omori ${ }^{24}$, M.J. Oreglia ${ }^{9}$, S. Orito ${ }^{24}$, M. Palazzo ${ }^{2}$, J. Pálinkás ${ }^{33, d}$, F.M. Palmonari ${ }^{2}$, J.P.Pansart ${ }^{21}$, G.Pásztor ${ }^{32}$, J.R.Pater ${ }^{16}$, G.N.Patrick ${ }^{20}$, M.J. Pearce ${ }^{1}$, S. Petzold ${ }^{27}$, J.E. Pilcher ${ }^{9}$, J. Pinfold ${ }^{30}$, D.E.Plane ${ }^{8}$, P.Poffenberger ${ }^{28}$, B. Poli ${ }^{2}$, A. Posthaus ${ }^{3}$,

H. Przysiezniak ${ }^{30}$, D.L. Rees ${ }^{1}$, D. Rigby ${ }^{1}$, M.G. Rison ${ }^{5}$, S.A. Robins ${ }^{13}$, N. Rodning ${ }^{30}$, J.M. Roney ${ }^{28}$, A. Rooke ${ }^{15}$, E. Ros ${ }^{8}$, A.M.Rossi ${ }^{2}$, M.Rosvick ${ }^{28}$, P. Routenburg ${ }^{30}$, Y. Rozen ${ }^{8}$, K. Runge ${ }^{10}$, O. Runolfsson ${ }^{8}$, D.R. Rust ${ }^{12}$, R. Rylko ${ }^{25}$, E.K.G.Sarkisyan ${ }^{23}$, M. Sasaki ${ }^{24}$, C. Sbarra ${ }^{2}$, A.D. Schaile ${ }^{8, e}$, O.Schaile ${ }^{10}$, F. Scharf ${ }^{3}$, P.Scharff-Hansen ${ }^{8}$, P.Schenk ${ }^{4}$, B.Schmitt ${ }^{3}$, M.Schröder ${ }^{8}$, H.C.Schultz-Coulon ${ }^{10}$, M.Schulz ${ }^{8}$, P.Schütz ${ }^{3}$, J.Schwiening ${ }^{3}$, W.G. Scott ${ }^{20}$, T.G. Shears ${ }^{16}$, B.C.Shen ${ }^{4}$, C.H. Shepherd-Themistocleous ${ }^{27}$, P. Sherwood ${ }^{15}$, G.P.Siroli ${ }^{2}$, A.Sittler ${ }^{27}$, A.Skillman ${ }^{15}$, A.Skuja ${ }^{17}$, A.M.Smith ${ }^{8}$, T.J. Smith ${ }^{28}$, G.A.Snow ${ }^{17}$, R.Sobie ${ }^{28}$, S.Söldner-Rembold ${ }^{10}$, R.W.Springer ${ }^{30}$, M.Sproston ${ }^{20}$, A.Stahl ${ }^{3}$, M.Starks ${ }^{12}$, K. Stephens ${ }^{16}$, J.Steuerer ${ }^{27}$, B.Stockhausen ${ }^{3}$, D. Strom ${ }^{19}$, F.Strumia ${ }^{8}$, P.Szymanski ${ }^{20}$,

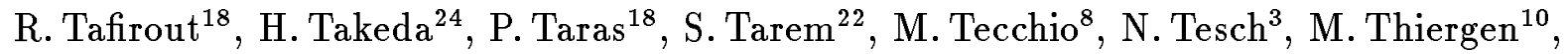
M.A. Thomson ${ }^{8}$, E. von Törne ${ }^{3}$, S. Towers ${ }^{6}$, M. Tscheulin ${ }^{10}$, E. Tsur ${ }^{23}$, A.S. Turcot ${ }^{9}$, 
M.F.Turner-Watson ${ }^{8}$, P. Utzat ${ }^{11}$, R. Van Kooten ${ }^{12}$, G. Vasseur ${ }^{21}$, M. Verzocchi ${ }^{10}$, P. Vikas ${ }^{18}$, M. Vincter ${ }^{28}$, E.H. Vokurka ${ }^{16}$, F. Wäckerle ${ }^{10}$, A. Wagner ${ }^{27}$, C.P. Ward ${ }^{5}$, D.R. Ward ${ }^{5}$, J.J. Ward ${ }^{15}$, P.M. Watkins ${ }^{1}$, A.T. Watson ${ }^{1}$, N.K. Watson ${ }^{7}$, P. Weber ${ }^{6}$, P.S. Wells ${ }^{8}$, N. Wermes ${ }^{3}$, J.S. White ${ }^{28}$, B. Wilkens ${ }^{10}$, G.W.Wilson ${ }^{27}$, J.A. Wilson ${ }^{1}$, T. Wlodek ${ }^{26}$, G. Wolf ${ }^{26}$, S. Wotton ${ }^{11}$, T.R. Wyatt ${ }^{16}$, S. Xella ${ }^{2}$, S. Yamashita ${ }^{24}$, G. Yekutieli ${ }^{26}$, K. Yoshimura $^{24}$, V. Zacek ${ }^{18}$,

${ }^{1}$ School of Physics and Space Research, University of Birmingham, Birmingham B15 2TT, UK ${ }^{2}$ Dipartimento di Fisica dell' Università di Bologna and INFN, I-40126 Bologna, Italy

${ }^{3}$ Physikalisches Institut, Universität Bonn, D-53115 Bonn, Germany

${ }^{4}$ Department of Physics, University of California, Riverside CA 92521, USA

${ }^{5}$ Cavendish Laboratory, Cambridge CB3 0HE, UK

${ }^{6}$ Ottawa-Carleton Institute for Physics, Department of Physics, Carleton University, Ottawa, Ontario K1S 5B6, Canada

${ }^{7}$ Centre for Research in Particle Physics, Carleton University, Ottawa, Ontario K1S 5B6, Canada

${ }^{8} \mathrm{CERN}$, European Organisation for Particle Physics, CH-1211 Geneva 23, Switzerland

${ }^{9}$ Enrico Fermi Institute and Department of Physics, University of Chicago, Chicago IL 60637, USA

${ }^{10}$ Fakultät für Physik, Albert Ludwigs Universität, D-79104 Freiburg, Germany

${ }^{11}$ Physikalisches Institut, Universität Heidelberg, D-69120 Heidelberg, Germany

${ }^{12}$ Indiana University, Department of Physics, Swain Hall West 117, Bloomington IN 47405, USA

${ }^{13}$ Queen Mary and Westfield College, University of London, London E1 4NS, UK

${ }^{14}$ Technische Hochschule Aachen, III Physikalisches Institut, Sommerfeldstrasse 26-28, D-52056

Aachen, Germany

${ }^{15}$ University College London, London WC1E 6BT, UK

${ }^{16}$ Department of Physics, Schuster Laboratory, The University, Manchester M13 9PL, UK

${ }^{17}$ Department of Physics, University of Maryland, College Park, MD 20742, USA

${ }^{18}$ Laboratoire de Physique Nucléaire, Université de Montréal, Montréal, Quebec H3C 3J7, Canada

${ }^{19}$ University of Oregon, Department of Physics, Eugene OR 97403, USA

${ }^{20}$ Rutherford Appleton Laboratory, Chilton, Didcot, Oxfordshire OX11 0QX, UK

${ }^{21}$ CEA, DAPNIA/SPP, CE-Saclay, F-91191 Gif-sur-Yvette, France

${ }^{22}$ Department of Physics, Technion-Israel Institute of Technology, Haifa 32000, Israel

${ }^{23}$ Department of Physics and Astronomy, Tel Aviv University, Tel Aviv 69978, Israel

${ }^{24}$ International Centre for Elementary Particle Physics and Department of Physics, University of Tokyo, Tokyo 113, and Kobe University, Kobe 657, Japan

${ }^{25}$ Brunel University, Uxbridge, Middlesex UB8 3PH, UK

${ }^{26}$ Particle Physics Department, Weizmann Institute of Science, Rehovot 76100, Israel

${ }^{27}$ Universität Hamburg/DESY, II Institut für Experimental Physik, Notkestrasse 85, D-22607

Hamburg, Germany

${ }^{28}$ University of Victoria, Department of Physics, P O Box 3055, Victoria BC V8W 3P6, Canada

${ }^{29}$ University of British Columbia, Department of Physics, Vancouver BC V6T 1Z1, Canada

${ }^{30}$ University of Alberta, Department of Physics, Edmonton AB T6G 2J1, Canada

${ }^{31}$ Duke University, Dept of Physics, Durham, NC 27708-0305, USA

${ }^{32}$ Research Institute for Particle and Nuclear Physics, H-1525 Budapest, P O Box 49, Hungary 
${ }^{33}$ Institute of Nuclear Research, H-4001 Debrecen, P O Box 51, Hungary

${ }^{a}$ and at TRIUMF, Vancouver, Canada V6T 2A3

${ }^{b}$ and Royal Society University Research Fellow

${ }^{c}$ and Institute of Nuclear Research, Debrecen, Hungary

${ }^{d}$ and Depart of Experimental Physics, Lajos Kossuth University, Debrecen, Hungary

${ }^{e}$ and Ludwig-Maximilians-Universität, München, Germany 


\section{Introduction}

In November 1995 the LEP $\mathrm{e}^{+} \mathrm{e}^{-}$collider at CERN was run for the first time well above the $\mathrm{Z}$ peak at centre-of-mass energies $(\sqrt{s})$ of 130-140 GeV (LEP1.5). This provided an opportunity to search for new particles at these higher energies, but below the $\mathrm{e}^{+} \mathrm{e}^{-} \rightarrow \mathrm{W}^{+} \mathrm{W}^{-}$threshold.

It is particularly interesting to search for charginos and neutralinos predicted by supersymmetric (SUSY) theories [1]. Charginos $\tilde{\chi}_{j}^{ \pm}$are the two mass eigenstates formed by the mixing of the fermionic partners of the charged gauge bosons (winos) and those of the charged Higgs bosons. Fermionic partners of the $\gamma$ (photinos), the $\mathrm{Z}$ boson (zinos), and the neutral Higgs bosons mix into the four mass eigenstates called neutralinos $\tilde{\chi}_{i}^{0} \cdot{ }^{1}$ The lightest neutralino $\tilde{\chi}_{1}^{0}$ is assumed to be the lightest supersymmetric particle and is therefore stable and invisible if R-parity [2] is conserved. We used the Minimal Supersymmetric Standard Model (MSSM) [3] to guide the analysis but more general cases were also studied.

If charginos are light enough, they can be pair-produced in $\mathrm{e}^{+} \mathrm{e}^{-}$collisions through $\gamma$ or $\mathrm{Z}$ exchange in the $s$-channel and through sneutrino $(\tilde{\nu})$ exchange in the $t$-channel. The production cross section is fairly large unless the sneutrino is light in which case destructive interference may occur between the $s$-channel and $t$-channel diagrams $[4,5]$.

The details of chargino decay depend on the parameters of the mixing and the masses of the scalar partners of the ordinary fermions $[4,5]$. The lightest chargino $\tilde{\chi}_{1}^{+}$can decay into a neutralino $\tilde{\chi}_{1}^{0}$ and a lepton pair: $\tilde{\chi}_{1}^{+} \rightarrow \tilde{\chi}_{1}^{0} \ell^{+} \nu$, or a neutralino and a quark pair: $\tilde{\chi}_{1}^{+} \rightarrow \tilde{\chi}_{1}^{0} q \bar{q}^{\prime}$ through virtual $\mathrm{W}$, slepton $(\tilde{\ell})$, or scalar quark $(\tilde{\mathrm{q}})$ emission. The effects of the latter are ignored with the assumption that scalar quarks are very heavy. $\tilde{\chi}_{1}^{+}$decay via virtual $\mathrm{W}$ emission is dominant in most of the MSSM parameter space; however, if the $\tilde{\chi}_{1}^{+}$is almost a pure wino and the $\tilde{\chi}_{1}^{0}$ is almost a pure SUSY partner of the $U(1)$ gauge boson, the $\tilde{\chi}_{1}^{+}$decay via the W boson is suppressed and the dominant decay mode may be $\tilde{\chi}_{1}^{0} \ell^{+} \nu$ via a virtual $\tilde{\ell}$ or $\tilde{\nu}$. Due to the energy and momentum carried away by the invisible $\tilde{\chi}_{1}^{0}$, chargino events are characterised by large missing energy and transverse momentum imbalance. At $\sqrt{s}=130-136 \mathrm{GeV}$, which is far below the threshold for pair production of real W's or Z's, the backgrounds to chargino pair production are expected to be relatively small.

Previous searches at LEP 1, running at centre-of-mass energies near the $\mathrm{Z}$ peak, set lower limits on the mass of the charginos at around $M_{\mathrm{Z}} / 2$ using a combination of direct searches and $\mathrm{Z}$ width measurements [6]. Similar but more model-dependent limits were obtained by the CDF and D0 Collaborations at the Tevatron [7].

Neutralino pairs $\left(\tilde{\chi}_{i}^{0} \tilde{\chi}_{j}^{0}\right)$ can be produced through an $s$-channel virtual $\mathrm{Z}$ boson or by $t$ -

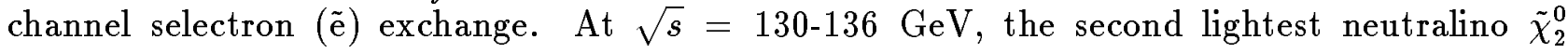
produced in conjunction with a $\tilde{\chi}_{1}^{0}$ could give the first direct signal for neutralinos, since single photon events from $\mathrm{e}^{+} \mathrm{e}^{-} \rightarrow \tilde{\chi}_{1}^{0} \tilde{\chi}_{1}^{0} \gamma$ suffer from background from $\mathrm{e}^{+} \mathrm{e}^{-} \rightarrow \nu \bar{\nu} \gamma$. At LEP 1 limits have been obtained from measurements of the width of the $\mathrm{Z}$ boson and direct searches [8].

If the $\tilde{\chi}_{2}^{0}$ is the lightest visible SUSY particle, it would decay into $\tilde{\chi}_{1}^{0} \ell^{+} \ell^{-}$or $\tilde{\chi}_{1}^{0} \mathrm{q} \bar{q}$ via a $\mathrm{Z}^{*}$, a Higgs boson, a scalar lepton, or a scalar quark. This leads to a similar experimental topology to that for chargino events.

In this paper we report on a direct search for charginos and neutralinos using the data collected with the OPAL detector at $\sqrt{s}=130-136 \mathrm{GeV}$. The data sample collected at $140 \mathrm{GeV}$ was very small and therefore was not used.

\footnotetext{
${ }^{1}$ The indices $i$ and $j$ are ordered by increasing mass.
} 


\section{The OPAL Detector and Event Simulation}

\subsection{The OPAL Detector}

The OPAL detector, which is described in detail in [9], is a multipurpose apparatus having nearly complete solid angle coverage. The central detector consists of a system of tracking chambers providing charged particle tracking over $96 \%$ of the full solid angle ${ }^{2}$ inside a $0.435 \mathrm{~T}$ solenoidal magnetic field. A lead-glass electromagnetic (EM) calorimeter located outside the magnet coil covers the full azimuthal range with excellent hermeticity in the polar angle range $|\cos \theta|<0.82$ for the barrel region and $0.81<|\cos \theta|<0.984$ for the endcap region. The magnet return yoke is instrumented for hadron calorimetry (HCAL) consisting of barrel and endcap sections along with pole tips that together cover the region $|\cos \theta|<0.99$. Calorimeters close to the beam axis measure the luminosity using small angle Bhabha scattering events and complete the geometrical acceptance down to $26 \mathrm{mrad}$. These include the forward detectors (FD) which are lead-scintillator sandwich calorimeters and at smaller angles, silicon tungsten calorimeters (SW) [10] located on both sides of the interaction point. The gap between the endcap EM calorimeter and FD is filled by an additional electromagnetic calorimeter, called the gamma-catcher.

\subsection{Monte Carlo Event Simulation}

The Monte Carlo generator SUSYGEN [11] was used to produce chargino and neutralino pair events. We also used a Monte Carlo generator similarly based on the calculation of the differential cross sections by Bartl et al. [4] for chargino pair production. The cross sections calculated by both generators agree very well. In both generators initial state radiation was included, and the JETSET7.4 package [12] was used for the hadronization of the quark-antiquark system in the chargino or neutralino hadronic decay with parameters specified in Ref. [13].

The most important parameters governing the chargino detection efficiency are the mass of the chargino $m_{\tilde{\chi}_{1}^{+}}$and the mass difference between the chargino and the lightest neutralino: $\Delta M_{+} \equiv\left(m_{\tilde{\chi}_{1}^{+}}-m_{\tilde{\chi}_{1}^{0}}\right)$. For the case of the neutralino, $m_{\tilde{\chi}_{2}^{0}}$ and $\Delta M_{0} \equiv\left(m_{\tilde{\chi}_{2}^{0}}-m_{\tilde{\chi}_{1}^{0}}\right)$ mainly determine the efficiency. The other important parameters are the decay branching fractions. We generated $\tilde{\chi}_{1}^{+} \tilde{\chi}_{1}^{-}$events at 30 points in the $m_{\tilde{\chi}_{1}^{+}} \Delta M_{+}$plane. For the $\tilde{\chi}_{1}^{0} \tilde{\chi}_{2}^{0}$ process, events were generated at 42 points in the $m_{\tilde{\chi}_{2}^{0}}-\Delta M_{0}$ plane.

There are several sources of background to the chargino and neutralino signals:

- Two photon processes are the most important background for the case of small $\Delta M_{+}$(or $\left.\Delta M_{0}\right)$, since signal events have small visible energy and small transverse momentum relative to the beam direction. We used the Monte Carlo generator PYTHIA [12] for generating events from two photon processes where the virtual mass $Q^{2}$ of both photons is smaller than $1.3 \mathrm{GeV}^{2}$ and the invariant mass of the photon-photon system $\left(M_{\gamma \gamma}\right)$ is greater than $3 \mathrm{GeV}$. For higher $Q^{2}$ events, the generator TWOGEN [14] was used. Event samples for all the possible processes (final state hadrons from point-like $\gamma \gamma \rightarrow q \bar{q}$ processes and from vector meson dominance, and all $\mathrm{e}^{+} \mathrm{e}^{-} \ell^{+} \ell^{-}$final states) were generated. Two photon events were not generated in the region

\footnotetext{
${ }^{2}$ A right-handed coordinate system is adopted, where the $x$-axis points to the centre of the LEP ring, and positive $z$ is along the electron beam direction. The angles $\theta$ and $\phi$ are the polar and azimuthal angles, respectively.
} 
$Q^{2}<1.3 \mathrm{GeV}^{2}$ and $M_{\gamma \gamma}<3 \mathrm{GeV}$. This region did not represent a serious background to the search presented here.

- $\tau$ pairs are the dominant background for the monojet signal when one of the $\tau$ 's decays into a very low momentum electron and two energetic neutrinos. $\tau^{+} \tau^{-} \gamma$ events are also a potential source of background for the topology of two acoplanar jets. The event generator KORALZ [15] was used for the generation of $\tau^{+} \tau^{-}(\gamma)$ events.

- Ordinary multijet hadronic events in which one or more jet momenta are mismeasured are the dominant background for the large mass difference case. The PYTHIA generator [12] was used to simulate hadronic events.

- Finally, four fermion processes in which at least one of the fermions is a neutrino constitute a serious background. Although the cross sections for these processes are small at $\sqrt{s}$ below the $\mathrm{W}^{+} \mathrm{W}^{-}$or $\mathrm{ZZ}$ threshold, their event topology is similar to that of the signal. Since the interference effects of many diagrams are important below the $\mathrm{W}^{+} \mathrm{W}^{-}$threshold, we used an event generator based on helicity amplitude calculations, which take into account all the relevant diagrams and interference effects [16]. The package also includes initial state photon radiation.

Generated signal and background events were processed through the full simulation of the OPAL detector [17], and the same event analysis chain was applied to simulated events as to the data.

\section{Analysis}

The present analysis is based on the data collected during the 1995 November run of LEP. Data used in this analysis corresponded to an integrated luminosity of $2.6 \mathrm{pb}^{-1}$ at a centre-of-mass energy of $\sqrt{s}=130 \mathrm{GeV}$ and $2.6 \mathrm{pb}^{-1}$ at $136 \mathrm{GeV}^{3}$.

The experimental signatures for $\tilde{\chi}_{1}^{+} \tilde{\chi}_{1}^{-}$or $\tilde{\chi}_{1}^{0} \tilde{\chi}_{2}^{0}$ events are an acoplanar pair of particles or jets, or a monojet topology with large transverse momentum with respect to the beam axis. If the mass difference between $\tilde{\chi}_{1}^{+}$and $\tilde{\chi}_{1}^{0}$ is as small as $5 \mathrm{GeV}$, the visible energy as well as the transverse momentum becomes small. The selection was designed to maintain reasonable efficiency for these cases.

Good charged tracks were selected using the same track quality requirements as in Ref. [18]. Tracks were required to have at least 20 measured space points, more than $50 \%$ of the hits geometrically expected, and transverse momentum exceeding $100 \mathrm{MeV}$. Electromagnetic clusters in the barrel region were required to have an energy of at least $170 \mathrm{MeV}$, and the clusters in the endcaps to have an energy of at least $250 \mathrm{MeV}$ and contain at least two adjacent lead glass blocks. Clusters in the hadron calorimeters were required to have an energy of at least $0.6 \mathrm{GeV}$ in the barrel and endcaps, and at least $2 \mathrm{GeV}$ in the pole tips.

The measurements of visible energy, mass and total transverse momentum of the events were performed by the method used in Ref. [18]. Four momentum vectors were formed for each track and calorimeter cluster, and then summed. The calorimeter clusters were treated as massless particles. In order to reduce the effects of double counting, four vectors based on the average expected energy deposition in the calorimeters for each charged track were then

\footnotetext{
${ }^{3}$ The actual centre-of-mass energies were estimated to be 130.26 and $136.23 \mathrm{GeV}$, but for the purpose of this analysis the nominal values of 130 and $136 \mathrm{GeV}$ were assumed.
} 
subtracted. This average energy deposition was parametrized as a function of track momentum and polar angle. The procedure used avoids the need for detailed matching between tracks and calorimeter clusters.

When calculating angles, such as the polar angle of the thrust axis, $\theta_{\text {thrust }}$, or the acoplanarity angle (defined below), the charged track momenta are more important than the calorimeter information due to their better angular resolution. The track momenta and momentum vectors of the EM or hadron calorimeter clusters not associated with charged tracks were first summed. In the case where a calorimeter cluster had associated good charged tracks, the scalar sum of the associated charged track momenta was subtracted from the cluster energy to reduce double counting. If the energy of a cluster was smaller than the scalar sum of the momenta of the associated tracks, the cluster energy was not used. If the number of charged particles in the event was equal to two, this method was also used to calculate all the variables including visible energy and invariant masses. These events are mainly lepton pairs while the algorithm of Ref. [18] was optimised for hadronic jets.

To select chargino and neutralino candidates, the number of good charged tracks was required to be at least two, and the ratio of the number of good tracks to the total number of tracks to be greater than 0.2 .

To reduce background from two photon processes and from multihadronic events where a jet axis is close to the beam direction, the total energy deposited in each silicon tungsten calorimeter had to be less than $5 \mathrm{GeV}$, less than $2 \mathrm{GeV}$ in each forward calorimeter, and less than $5 \mathrm{GeV}$ in each side of the gamma-catcher. In addition, the visible energy in the region of $|\cos \theta|>0.8$ should be less than $30 \%$ of the total visible energy (cut 1 ). After this cut the distribution of total visible energy normalised by $\sqrt{s}$ is shown in Fig. 1a and the distribution of event transverse momentum ( $P_{t}$, magnitude of the vector sum of transverse momentum components) measured without including the hadron calorimeter is shown in Fig. 1b. Since two photon Monte Carlo events were generated with $M_{\gamma \gamma}>3 \mathrm{GeV}$, the visible mass of the events was required to be larger than $3 \mathrm{GeV}$ just for these plots in order to demonstrate that the normalization of the two photon events agrees with the Monte Carlo prediction. The events with small invariant mass were eliminated from the data by demanding that the $P_{t}$ be greater than $4 \mathrm{GeV}$ and the event transverse momentum measured including the hadron calorimeter $\left(P_{t}^{\mathrm{HCAL}}\right)$ should be greater than $5 \mathrm{GeV}$ (cut 2). Although most of the events from two photon processes were rejected by the $P_{t}$ cut, the $P_{t}^{\mathrm{HCAL}}$ cut was applied to reject occasional two photon events with a high transverse momentum neutral hadron.

"Radiative return" events from $\mathrm{e}^{+} \mathrm{e}^{-} \rightarrow \mathrm{Z} \gamma$, where the $\gamma$ escaped down the beam pipe, were rejected by requiring that the polar angle of the missing momentum direction $\theta_{\text {miss }}$ satisfy $\left|\cos \theta_{\text {miss }}\right|<0.7$ (cut 3). Events with a photon of energy greater than $15 \mathrm{GeV}$ and accompanied by no good tracks (except for track pairs identified as photon conversions) within a cone of half angle $25^{\circ}$ from the photon direction were rejected (cut 4 ).

To remove a large fraction of the multihadron events the visible energy was required to be less than $0.7 \sqrt{s}$ (cut 5). Finally, events that were kinematically consistent with $\tau^{+} \tau^{-}(\gamma)$ were rejected (cut 6). This $\tau^{+} \tau^{-}(\gamma)$ rejection was accomplished by requirements on multiplicity, momentum sum and invariant mass of charged particles in each hemisphere of the event, and a requirement on the acoplanarity angle which depended on the absolute value and the polar angle of the charged particle momentum sum in each hemisphere.

The tracks and the clusters in an event were then divided into two hemispheres defined by the plane perpendicular to the thrust axis. If one of the hemispheres had an energy of less 
than $1 \mathrm{GeV}$ and also contained no good tracks, the event was categorised as a monojet event. Otherwise the event was classified as a dijet event. According to these criteria, ten events in the data were classified as dijet events, and four as monojet events.

For dijet events, the additional selection criteria below were applied. The polar angle of the event thrust axis was required to satisfy $\left|\cos \theta_{\text {thrust }}\right|<0.9$ (cut A1). No events were removed by this requirement, but it ensures good resolution of the acoplanarity angle. The acoplanarity angle $\phi_{\text {acop }}$ was defined as $\pi-\phi_{\text {open }}$, where $\phi_{\text {open }}$ is the azimuthal opening angle between the directions of the two momentum sums of the particles in the thrust hemispheres. The acoplanarity angle $\left(\phi_{\text {acop }}\right)$ between the momentum sums in the two hemispheres was required to be greater than $20^{\circ}$ (cut A2). The acoplanarity angle distribution just before the cut is shown in Fig. 1c. The expected distribution for $\tilde{\chi}_{1}^{+} \tilde{\chi}_{1}^{-}$with $m_{\tilde{\chi}_{1}^{+}}=60 \mathrm{GeV}$ and $m_{\tilde{\chi}_{1}^{0}}=30 \mathrm{GeV}$ is also shown.

For events classified as monojets, additional selection criteria were required. To reject $\mathrm{e}^{+} \mathrm{e}^{-} \rightarrow$ $\mathrm{e}^{+} \mathrm{e}^{-} \tau^{+} \tau^{-}$events where one of the $\tau^{\prime}$ 's decays into a soft electron and to reject four fermion processes $\mathrm{e}^{+} \mathrm{e}^{-} \rightarrow \mathrm{Z}^{*} \gamma^{*} \rightarrow \nu \bar{\nu} \mathrm{f} \bar{f}$, where $\mathrm{f}$ is a charged lepton or a quark, the visible mass of the event was required to be greater than $2 \mathrm{GeV}$ (cut B1). The visible mass distribution just before the cut is shown in Fig. $1 \mathrm{~d}$. The expected distribution for $\tilde{\chi}_{1}^{0} \tilde{\chi}_{2}^{0}$ with $m_{\tilde{\chi}_{2}^{0}}=80 \mathrm{GeV}$ and $m_{\tilde{\chi}_{1}^{0}}=30 \mathrm{GeV}$ is also shown. Furthermore, the ratio of the visible mass to the visible energy $\left(M_{\mathrm{vis}} / E_{\mathrm{vis}}\right)$ was required to be larger than 0.4 (cut B2).

No events were observed in the data after the above cuts.

The remaining numbers of events after each cut are listed in Table 1. For comparison the table also shows the corresponding numbers of simulated events for background processes and for three samples of simulated $\tilde{\chi}_{1}^{+} \tilde{\chi}_{1}^{-}$and $\tilde{\chi}_{1}^{0} \tilde{\chi}_{2}^{0}$ events.

The efficiency for $\tilde{\chi}_{1}^{+} \tilde{\chi}_{1}^{-}$events is about $42 \%$ for $m_{\tilde{\chi}_{1}^{+}}=60 \mathrm{GeV}$ and $m_{\tilde{\chi}_{1}^{0}}=30 \mathrm{GeV}$ at $\sqrt{s}=130 \mathrm{GeV}$ for a $\tilde{\chi}^{+}$decay via a virtual $\mathrm{W}$. The efficiency decreases to $15 \%$ for $m_{\tilde{\chi}_{1}^{0}}=$ $55 \mathrm{GeV}$. For the case of purely leptonic decays, the efficiencies are about $5 \%$ lower than for the decay via $\mathrm{W}^{*}$ for the same $\tilde{\chi}_{1}^{+}$and $\tilde{\chi}_{1}^{0}$ mass combination. The efficiency for $\tilde{\chi}_{1}^{0} \tilde{\chi}_{2}^{0}$ events is typically $30-40 \%$ over a wide range of $\tilde{\chi}_{2}^{0}$ and $\tilde{\chi}_{1}^{0}$ masses. The efficiency drops to $10 \%$ for the small mass difference of $\Delta M_{0}=5 \mathrm{GeV}$. For the extraction of limits described in the following section the efficiency at an arbitrary point of $m_{\tilde{\chi}_{1}^{+}}\left(m_{\tilde{\chi}_{2}^{0}}\right)$ and $\Delta M_{+}\left(\Delta M_{0}\right)$ was interpolated using a polynomial fit to the efficiencies determined using Monte Carlo simulations.

\section{Results}

No evidence for chargino or neutralino production is observed in the data; therefore, limits are calculated. In the first analysis, upper limits on the cross sections as functions of chargino and neutralino masses were calculated for $100 \%$ branching fractions into specific decay modes (model independent analysis). In the second analysis, limits on chargino and neutralino masses were calculated within the framework of the MSSM, and limits in the MSSM parameter space were obtained. 


\begin{tabular}{|l||r||r||r|r|r|r||r|r|r|}
\hline & data & $\begin{array}{r}\text { total } \\
\text { bkg. }\end{array}$ & $\mathrm{q} \overline{\mathrm{q}}(\gamma)$ & $\tau \tau(\gamma)$ & ${ }^{\prime} \gamma \gamma^{\prime}$ & $4-\mathrm{f}$ & $\tilde{\chi}_{1}^{+} \tilde{\chi}_{1}^{-}$ & $\tilde{\chi}_{1}^{+} \tilde{\chi}_{1}^{-}$ & $\tilde{\chi}_{1}^{0} \tilde{\chi}_{2}^{0}$ \\
\hline$m_{\tilde{\chi}_{1}^{ \pm}}(\mathrm{GeV})$ & & & & & & & 60 & 60 & - \\
$m_{\tilde{\chi}_{1}^{0}}(\mathrm{GeV})$ & & & & & & & 55 & 30 & 30 \\
$m_{\tilde{\chi}_{2}^{0}}(\mathrm{GeV})$ & & & & & & & - & - & 80 \\
\hline no cuts & - & - & 1650 & 115.3 & $105 \mathrm{k}$ & 11.1 & 1000 & 1000 & 1000 \\
cut (1) & 37910 & 8896 & 797.9 & 58.6 & 7104 & 2.8 & 680 & 733 & 758 \\
cut (2) & 511 & 546.2 & 352.3 & 44.8 & 8.0 & 2.5 & 180 & 677 & 746 \\
cut (3) & 207 & 245.8 & 162.5 & 30.5 & 3.1 & 2.0 & 176 & 556 & 632 \\
cut (4) & 169 & 206.3 & 132.6 & 26.7 & 3.1 & 2.0 & 176 & 556 & 632 \\
cut (5) & 35 & 34.5 & 6.8 & 22.3 & 2.9 & 1.9 & 176 & 556 & 631 \\
cut (6) & 14 & 16.0 & 6.8 & 4.2 & 2.9 & 1.9 & 176 & 554 & 631 \\
\hline dijet & 10 & 13.7 & 6.8 & 4.2 & 2.1 & $\mathbf{0 . 4 2}$ & 104 & 483 & 233 \\
cut (A1) & 10 & 13.7 & 6.8 & 4.2 & 2.1 & $\mathbf{0 . 4 0}$ & 103 & 474 & 233 \\
cut (A2) & $\mathbf{0}$ & $\mathbf{0 . 7 5}$ & $\mathbf{0 . 0}$ & $\mathbf{0 . 1 7}$ & $\mathbf{0 . 2 6}$ & $\mathbf{0 . 3 3}$ & 91 & 366 & 191 \\
\hline monojet & 4 & 2.31 & $\mathbf{0 . 0}$ & $\mathbf{0 . 0 3}$ & $\mathbf{0 . 7 8}$ & 1.50 & 72 & 71 & 398 \\
cut (B1) & $\mathbf{0}$ & $\mathbf{0 . 8 8}$ & $\mathbf{0 . 0}$ & $\mathbf{0 . 0 2}$ & $\mathbf{0 . 0 0}$ & $\mathbf{0 . 8 5}$ & 70 & 71 & 382 \\
cut (B2) & $\mathbf{0}$ & $\mathbf{0 . 1 8}$ & $\mathbf{0 . 0}$ & $\mathbf{0 . 0 1}$ & $\mathbf{0 . 0 0}$ & $\mathbf{0 . 1 7}$ & 65 & 52 & 90 \\
\hline \hline (A2+B2) & $\mathbf{0}$ & $\mathbf{0 . 9 3}$ & $\mathbf{0 . 0}$ & $\mathbf{0 . 1 8}$ & $\mathbf{0 . 2 6}$ & $\mathbf{0 . 4 9}$ & 156 & 418 & 281 \\
\hline
\end{tabular}

Table 1: The remaining numbers of events normalised to the integrated luminosity of the data for various background processes are compared with data after each cut. Numbers for three simulated event samples of $\tilde{\chi}_{1}^{+} \tilde{\chi}_{1}^{-}$with $\tilde{\chi}_{1}^{+} \rightarrow \tilde{\chi}_{1}^{0} \mathrm{~W}^{*}$, and $\tilde{\chi}_{1}^{0} \tilde{\chi}_{2}^{0}$ with $\tilde{\chi}_{2}^{0} \rightarrow$ $\tilde{\chi}_{1}^{0} \mathrm{Z}^{*}$ are also given (starting from 1000 events for each). The numbers given after cuts (A1) or (A2) are for dijet events only, and the numbers after cuts (B1) or (B2) are for monojet events only. The numbers of events expected from two photon processes do not include the region $M_{\gamma \gamma}<3 \mathrm{GeV}$ with $Q^{2}<1.3 \mathrm{GeV}^{2}$.

\subsection{Systematic Errors}

The common systematic errors for the model independent analysis and the analysis within the framework of the MSSM were first considered. The systematic error on the integrated luminosity was $1.5 \%$, which was evaluated from half the difference between the luminosities measured by the FD detector and the SW detector. The relative errors on the signal efficiencies due to Monte Carlo statistics and the interpolation errors at an arbitrary point of $m_{\tilde{\chi}_{1}^{+}}\left(m_{\tilde{\chi}_{2}^{0}}\right)$ and $m_{\tilde{\chi}_{1}^{0}}$ were evaluated to be $2-10 \%$ depending on the combination of the masses and the branching fractions. The systematic error due to the trigger efficiency was negligible for the selected signal events.

The various systematic errors were summed quadratically and subtracted from the signal efficiency.

\subsection{Limits on the Production Cross Sections}

We obtain upper limits on the production cross sections at the $95 \%$ confidence level (C.L.) for $\tilde{\chi}_{1}^{+} \tilde{\chi}_{1}^{-}$and $\tilde{\chi}_{1}^{0} \tilde{\chi}_{2}^{0}$ assuming specific decay modes. These limits do not depend on the details of the SUSY models. The contours of the upper limits for the $\tilde{\chi}_{1}^{+} \tilde{\chi}_{1}^{-}$cross sections at $\sqrt{s}=136 \mathrm{GeV}$ 
are shown in Fig. 2, assuming $\tilde{\chi}_{1}^{+} \rightarrow \tilde{\chi}_{1}^{0} \mathrm{~W}^{*}$ with $100 \%$ branching fraction (Fig. 2a) or $\tilde{\chi}_{1}^{+} \rightarrow$ $\tilde{\chi}_{1}^{0} \ell^{+} \nu$ via $\tilde{\ell}$ or $\tilde{\nu}$ with $100 \%$ branching fraction (Fig. $2 \mathrm{~b}$ ). The cross sections at $\sqrt{s}=130 \mathrm{GeV}$ were assumed to be the same as at $136 \mathrm{GeV}$ except if $2 m_{\tilde{\chi}_{1}^{+}}>130 \mathrm{GeV}$. Similarly, the contours of the upper limit for the $\tilde{\chi}_{1}^{0} \tilde{\chi}_{2}^{0}$ cross sections are shown for $\tilde{\chi}_{2}^{0} \rightarrow \tilde{\chi}_{1}^{0} \mathrm{Z}^{*}$ with $100 \%$ branching fraction (Fig. 2c), or $\tilde{\chi}_{2}^{0} \rightarrow \tilde{\chi}_{1}^{0} \ell^{+} \ell^{-}$via $\tilde{\ell}$ with $100 \%$ decay branching fraction (Fig. 2d). The Standard Model branching fractions were used for the $\mathrm{W}^{*}$ and $\mathrm{Z}^{*}$ decays including the invisible decay mode $\mathrm{Z}^{*} \rightarrow \nu \bar{\nu}$.

If the cross section for $\tilde{\chi}_{1}^{+} \tilde{\chi}_{1}^{-}$is larger than $3.5 \mathrm{pb}$, we can exclude the $\tilde{\chi}_{1}^{+}$for masses up to $65.0 \mathrm{GeV}$ at the $95 \%$ C.L. for $\Delta M_{+} \geq 10 \mathrm{GeV}$ independent of the decay mode, and almost up to the kinematical boundary for $\tilde{\chi}_{1}^{+}$decay via $W^{*}$. From Fig. 2c, we can also exclude the $\tilde{\chi}_{2}^{0}$ for masses almost up to the kinematical boundary of $\left(m_{\tilde{\chi}_{1}^{0}}+m_{\tilde{\chi}_{2}^{0}}\right)<\sqrt{s}$ at the 95\% C.L. for $\Delta M_{0} \geq 13 \mathrm{GeV}$, if the cross section for $\tilde{\chi}_{1}^{0} \tilde{\chi}_{2}^{0}$ with $\tilde{\chi}_{2}^{0} \rightarrow \tilde{\chi}_{1}^{0} Z^{*}$ is larger than $6 \mathrm{pb}$. The region $\left(m_{\tilde{\chi}_{1}^{0}}+m_{\tilde{\chi}_{2}^{0}}\right)<M_{\mathrm{Z}}$, which is accessible at LEP 1 , is not considered in this analysis. For $\tilde{\chi}_{1}^{0} \tilde{\chi}_{2}^{0}$ produced through $\mathrm{Z}$ boson decay better limits were obtained at LEP 1 [8].

\subsection{Limits on the Minimal Supersymmetric Standard Model}

The negative results of the above searches can be interpreted in the framework of the MSSM where the gaugino-higgsino sector of the theory is completely determined by three parameters: $M_{2}$, the $S U(2)$ gaugino mass at the weak scale; $\mu$, the mass coupling strength between the two Higgs superfields; and $\tan \beta$, the ratio $\left\langle v_{2}\right\rangle /\left\langle v_{1}\right\rangle$ of the vacuum expectation values of the two Higgs doublets. For a given set of these parameters there are, at tree level, unique relations [11] that determine masses and coupling constants of all gauginos. The scanned regions of the parameters were $0 \leq M_{2} \leq 1500 \mathrm{GeV},-400 \leq \mu \leq 400 \mathrm{GeV}$, for two values of $\tan \beta: \tan \beta=$ 1.5, a small value interesting for infrared fixed-point predictions for the top quark [19], and $\tan \beta=35$, approximately equal to the ratio of the top quark mass to the $b$ quark mass favoured by Yukawa coupling unification at a large mass scale [20]. A more general case of all values of $\tan \beta>1$ was also considered. The scanned ranges of $M_{2}$ and $\mu$ were checked to be large enough so that the exclusion regions presented change negligibly for larger ranges.

The $\tilde{\mathrm{f}}$ spectrum is further constrained by assuming soft symmetry breaking at the Grand Unification scale (GUT) with a common mass scale $m_{0}$ (defined at the GUT scale) [21]. A light $m_{0}$ sets the masses of the $\tilde{\nu}$ and $\tilde{\ell}$ to low values, thereby enhancing $t$-channel exchange diagrams that may have destructive interference with $s$-channel diagrams reducing the cross section for chargino production. Small values of $m_{0}$ also tend to enhance the leptonic branching ratio of charginos. On the other hand, fixing $m_{0}$ to high values decouples the $\tilde{\nu}$ and $\tilde{\mathbf{e}}$ from the theory, thereby enhancing the chargino production rate. We therefore present results here in the two scenarios: $m_{0}=1 \mathrm{TeV}$, and the other extreme, the smallest $m_{0}$ consistent with light $\tilde{\ell}$ and $\tilde{\nu}$ not yet excluded by LEP 1 , namely $m_{\tilde{\ell}}>45.0 \mathrm{GeV}$ and $m_{\tilde{\nu}}>41.8 \mathrm{GeV}[22]$.

In the MSSM, production cross sections and decay branching fractions of charginos and neutralinos are determined by the four SUSY parameters; $M_{2}, \mu, \tan \beta$ and $m_{0}$. In regions where chargino production is suppressed, SUSY signatures can be explored via neutralino production. The analysis presented here is therefore based on combining both the neutralino and chargino searches into one analysis. At each point in the MSSM parameter space the number of events expected for $\tilde{\chi}_{1}^{+} \tilde{\chi}_{1}^{-}, \tilde{\chi}_{1}^{0} \tilde{\chi}_{2}^{0}$, as well as $\tilde{\chi}_{2}^{0} \tilde{\chi}_{2}^{0}$ were added. The decay branching fractions were calculated and the corresponding detection efficiencies were used in order to set exclusion limits 
at the $95 \%$ C.L. in three different MSSM parameter planes. Since $\tilde{\chi}_{2}^{0}$ can decay into $\tilde{\chi}_{1}^{0}$ and the lightest Higgs boson, the efficiency for this case was checked and found to be similar to that for the $\tilde{\chi}_{2}^{0} \rightarrow \tilde{\chi}_{1}^{0} \mathrm{q} \overline{\mathrm{q}}$ decay via a $\mathrm{Z}^{*}$.

The $\tilde{\chi}_{2}^{0}$ can decay into $\nu \bar{\nu} \tilde{\chi}_{1}^{0}$ via a $\mathrm{Z}^{*}$ or $\tilde{\chi}_{1}^{0} \tilde{\chi}_{1}^{0} \tilde{\chi}_{1}^{0}$ via the lightest Higgs, leading to invisible events. In most of the MSSM parameter space the $\tilde{\chi}_{1}^{+}$is lighter than the $\tilde{\chi}_{2}^{0}$. Therefore the cascade decay of $\tilde{\chi}_{2}^{0} \rightarrow \tilde{\chi}_{1}^{ \pm} q \bar{q}^{\prime}$ or $\rightarrow \tilde{\chi}_{1}^{ \pm} \ell^{\mp} \nu$ with subsequent decay of the $\tilde{\chi}_{1}^{ \pm}$is possible. These effects were taken into account in calculating the limits.

The results are shown in Figs. 3 and 4 . Figure 3 shows the exclusion contours in the $M_{2}-\mu$ plane for the case $m_{0}=1 \mathrm{TeV}$ (Fig. 3a) and a light $m_{0}$ (Fig. $3 \mathrm{~b}$ ) for $\tan \beta=1.5$. The same plots are shown for $\tan \beta=35$ (Fig. $3 \mathrm{c}$ and $3 \mathrm{~d}$ ). Most of the excluded regions are due to the limits from the $\tilde{\chi}_{1}^{+} \tilde{\chi}_{1}^{-}$search. The $\tilde{\chi}_{1}^{0} \tilde{\chi}_{2}^{0}$ signal contributes only in the limited area near the kinematical boundary of the large $M_{2}$ region.

Figure 4 shows the region excluded at $95 \%$ C.L. in the $m_{\tilde{\chi}_{1}^{0}-} m_{\tilde{\chi}_{1}^{+}}$plane. The limits are shown for the combinations of two $m_{0}$ values and two $\tan \beta$ values. Similar exclusion regions in the $m_{\tilde{\chi}_{1}^{0}}-m_{\tilde{\chi}_{2}^{0}}$ plane are also shown in Fig. 4 . Again, most of the excluded regions are due to the limits from the $\tilde{\chi}_{1}^{+} \tilde{\chi}_{1}^{-}$search.

Figure $4 \mathrm{e}$ and $4 \mathrm{f}$ show the regions excluded at $95 \%$ C.L. in the $m_{\tilde{\chi}_{1}^{0}}-m_{\tilde{\chi}_{1}^{+}}$plane assuming $m_{0}=1 \mathrm{TeV}$ or the minimum $m_{0}$. The exclusion regions were found by scanning through $\tan \beta$ $(\tan \beta>1)$ to find the smallest number of events expected ( $\operatorname{sum}$ of $\tilde{\chi}_{1}^{+} \tilde{\chi}_{1}^{-}, \tilde{\chi}_{1}^{0} \tilde{\chi}_{2}^{0}$ and $\tilde{\chi}_{2}^{0} \tilde{\chi}_{2}^{0}$ events after all the cuts) at any point of the $M_{2}-\mu$ plane and for any value of $\tan \beta$ considered.

From the above plots one can set lower limits at 95\% C.L. on the chargino and neutralino masses as given in Table 2. Definitive lower limits for neutralino masses are not possible when considering the entire region $\tan \beta>1$ since for values of $\tan \beta$ close to 1 , typical considered mass values of $\tilde{\chi}_{1}^{0}$ and $\tilde{\chi}_{2}^{0}$ can result from MSSM parameters that give chargino masses that are not kinematically accessible for $\sqrt{s}=130-136 \mathrm{GeV}$.

\begin{tabular}{|c||c|c|c|c|c|}
\hline \multirow{2}{*}{ Mass } & \multicolumn{2}{|c|}{$\tan \beta=1.5$} & \multicolumn{2}{c|}{$\tan \beta=35$} & $\tan \beta>1$ \\
\cline { 2 - 6 } & Min. $m_{\mathbf{0}}$ & $m_{0}=1 \mathrm{TeV}$ & Min. $m_{\mathbf{0}}$ & $m_{\mathbf{0}}=1 \mathrm{TeV}$ & $m_{\mathbf{0}}=1 \mathrm{TeV}$ \\
\hline$m_{\tilde{\chi}_{1}^{+}}(\mathrm{GeV})$ & $>60.7$ & $>65.4$ & $>58.7$ & $>65.6$ & $>63.9$ \\
$m_{\tilde{\chi}_{1}^{0}}(\mathrm{GeV})$ & $>12.0$ & $>21.4$ & $>32.0$ & $>35.2$ & - \\
$m_{\tilde{\chi}_{2}^{0}}(\mathrm{GeV})$ & $>45.3$ & $>47.5$ & $>61.0$ & $>67.5$ & - \\
\hline
\end{tabular}

Table 2: Lower limits at $95 \%$ C.L. on the chargino mass $m_{\tilde{\chi}_{1}^{+}}$, the lightest neutralino mass $m_{\tilde{\chi}_{1}^{0}}$, and the second lightest neutralino mass $m_{\tilde{\chi}_{2}^{0}}$. These limits are given for the smallest $m_{0}$ possible to comply with the LEP $1 \tilde{\ell}$ and $\tilde{\nu}$ limits and also for $m_{0}=1 \mathrm{TeV}$ with the mass-difference conditions $\Delta M_{+} \geq 10 \mathrm{GeV}$ and $\Delta M_{0} \geq 10 \mathrm{GeV}$.

\section{Summary and Conclusion}

We have analyzed a data sample corresponding to an integrated luminosity of $2.6 \mathrm{pb}^{-1}$ at $\sqrt{s}=130 \mathrm{GeV}$ and $2.6 \mathrm{pb}^{-1}$ at $136 \mathrm{GeV}$ collected with the OPAL detector to search for pair production of charginos and neutralinos predicted by supersymmetric theories. No events remained after the selection cuts. This is consistent with the expected background of 0.9 events. 
Within the framework of MSSM and for the conditions $\Delta M_{+} \geq 10 \mathrm{GeV}$ and $\Delta M_{0} \geq 10 \mathrm{GeV}$ one can set lower limits at $95 \%$ C.L. on the chargino and neutralino masses: $m_{\tilde{\chi}_{1}^{0}}>21.4 \mathrm{GeV}$, $m_{\tilde{\chi}_{2}^{0}}>47.5 \mathrm{GeV}$ and $m_{\tilde{\chi}_{1}^{+}}>65.4 \mathrm{GeV}$ for $\tan \beta=1.5 . m_{\tilde{\chi}_{1}^{0}}>35.2 \mathrm{GeV}, m_{\tilde{\chi}_{2}^{0}}>67.5 \mathrm{GeV}$ and $m_{\tilde{\chi}_{1}^{+}}>65.6 \mathrm{GeV}$ are the limits obtained for $\tan \beta=35$. These limits were obtained for $m_{0}=1 \mathrm{TeV}$. For the smallest $m_{0}$ possible to comply with the LEP $1 \tilde{\ell}$ and $\tilde{\nu}$ limits, the mass limits are reduced by at most $7 \mathrm{GeV}$ for the chargino case.

\section{Acknowledgements}

It is a pleasure to thank the SL Division for the efficient operation of the LEP accelerator at the new energies of $\sqrt{s}=130-140 \mathrm{GeV}$ with good beam conditions and for their continuing close cooperation with our experimental group. In addition to the support staff at our own institutions we are pleased to acknowledge the

Department of Energy, USA,

National Science Foundation, USA,

Particle Physics and Astronomy Research Council, UK,

Natural Sciences and Engineering Research Council, Canada,

Fussefeld Foundation,

Israel Ministry of Science,

Israel Science Foundation, administered by the Israel Academy of Science and Humanities,

Minerva Gesellschaft,

Japanese Ministry of Education, Science, Sports and Culture (the Monbusho) and a grant un-

der the Monbusho International Science Research Program,

German Israeli Bi-national Science Foundation (GIF),

Direction des Sciences de la Matière du Commissariat à l'Energie Atomique, France, Bundesministerium für Bildung, Wissenschaft, Forschung und Technologie, Germany, National Research Council of Canada, A.P. Sloan Foundation, and the

Hungarian Foundation for Scientific Research, OTKA T-016660.

\section{References}

[1] Y. Gol'fand and E. Likhtam, JETP Lett. 13 (1971) 323;

D. Volkov and V. Akulov, Phys. Lett. B46 (1973) 109;

J. Wess and B. Zumino, Nucl. Phys. B70 (1974) 39.

[2] P. Fayet, Unification of the Fundamental Particle Interactions, Plenum Press (1980), p. 587.

[3] H. P. Nilles, Phys. Rep. 110 (1984) 1;

H. E. Haber and G. L. Kane, Phys. Rep. 117 (1985) 75.

[4] A. Bartl, H. Fraas and W. Majerotto, Z. Phys. C30 (1986) 441;

A. Bartl, H. Fraas and W. Majerotto, Z. Phys. C41 (1988) 475;

A. Bartl, H. Fraas, W. Majerotto and B. Mösslacher, Z. Phys. C55 (1992) 257. 
[5] M. Chen, C. Dionisi, M. Martinez and X. Tata, Phys. Rep. 159 (1988) 201;

J. L. Feng and M. J. Strassler, Phys. Rev. D51 (1995) 4661.

[6] L3 Collab., B. Adeva et al., Phys. Lett. B233 (1989) 530;

ALEPH Collab., D. Decamp et al., Phys. Lett. B236 (1990) 86;

OPAL Collab., M.Z. Akrawy et al., Phys. Lett. B240 (1990) 261;

DELPHI Collab., P. Abreu et al., Phys. Lett. B247 (1990) 157;

ALEPH Collab., D. Decamp et al., Phys. Rep. 216 (1992) 253.

[7] CDF Collab., J. Hauser, Searches for SUSY by CDF, in the Proc. of the 10th Topical Workshop on Proton-Antiproton Collider Physics, Fermilab, IL, USA, May 1995;

D0 Collab., S. Abachi et al., Search for $\tilde{W}_{1} \tilde{Z}_{2}$ Production via Trilepton Final States in p $\bar{p}$ Collisions at $\sqrt{s}=1.8 \mathrm{TeV}$, FERMILAB Pub-95/385-E, submitted to Phys. Rev. Lett.

[8] OPAL Collab., G. Alexander et al., Topological Search for the Production of Neutralinos and Scalar Particles, CERN-PPE/96-019, submitted to Phys. Lett.

[9] OPAL Collab., K. Ahmet et al., Nucl. Instr. Meth. A305 (1991) 275.

[10] B.E. Anderson et al., IEEE Transactions on Nuclear Science 41 (1994) 845.

[11] S. Katsanevas and S. Melanchroinos, to be published in the LEP200 workshop report, CERN 1995. The formulae found in the following references are employed:

Ref. 4 and A. Bartl, H. Fraas, and W. Majerotto, Nucl. Phys. B278 (1986) 1, and Z. Phys. C34 (1987) 411.

[12] T. Sjöstrand, Comp. Phys. Comm. 39 (1986) 347;

T. Sjöstrand, PYTHIA 5.7 and JETSET7.4 Manual, CERN-TH 7112/93.

[13] OPAL Collab., G. Alexander et al., CERN PPE/95-126, to be published in Z. Phys.

[14] A. Buijs et al., Comp. Phys. Comm. 79 (1994) 523.

[15] S. Jadach, B. F. L. Ward, Z. Wa̧s, Comp. Phys. Comm. 79 (1994) 503.

[16] The calculation of the cross section is based on the method described in the preprint by H. Murayama, I. Watanabe and K. Hagiwara, KEK Report 91-11 (1991). The initial state radiation is implemented according to a formula in F. A. Berends, R. Pittau and R. Kleiss, Nucl. Phys. B426 (1994) 344.

[17] J. Allison et al., Nucl. Instr. Meth. A317 (1992) 47.

[18] OPAL Collab., M.Z. Akrawy et al., Phys. Lett. B253 (1991) 511.

In the present analysis an improved parametrization of the energy measurement was used.

[19] M. Carena and C.E.M. Wagner, Nucl. Phys. B452 (1995) 45.

[20] J. Ellis, S. Kelley, and D.V. Nanopoulos, Phys. Lett. B287 (1992) 95.

[21] M. Drees and M. M. Nojiri, Nucl. Phys. B369 (1992) 54.

M. Carena, S. Dimopolous, S. Raby and C.E.M. Wagner, Phys. Rev. D52 (1995) 4133.

[22] Particle Data Group, Phys. Rev. D50 (1994) 1173. 

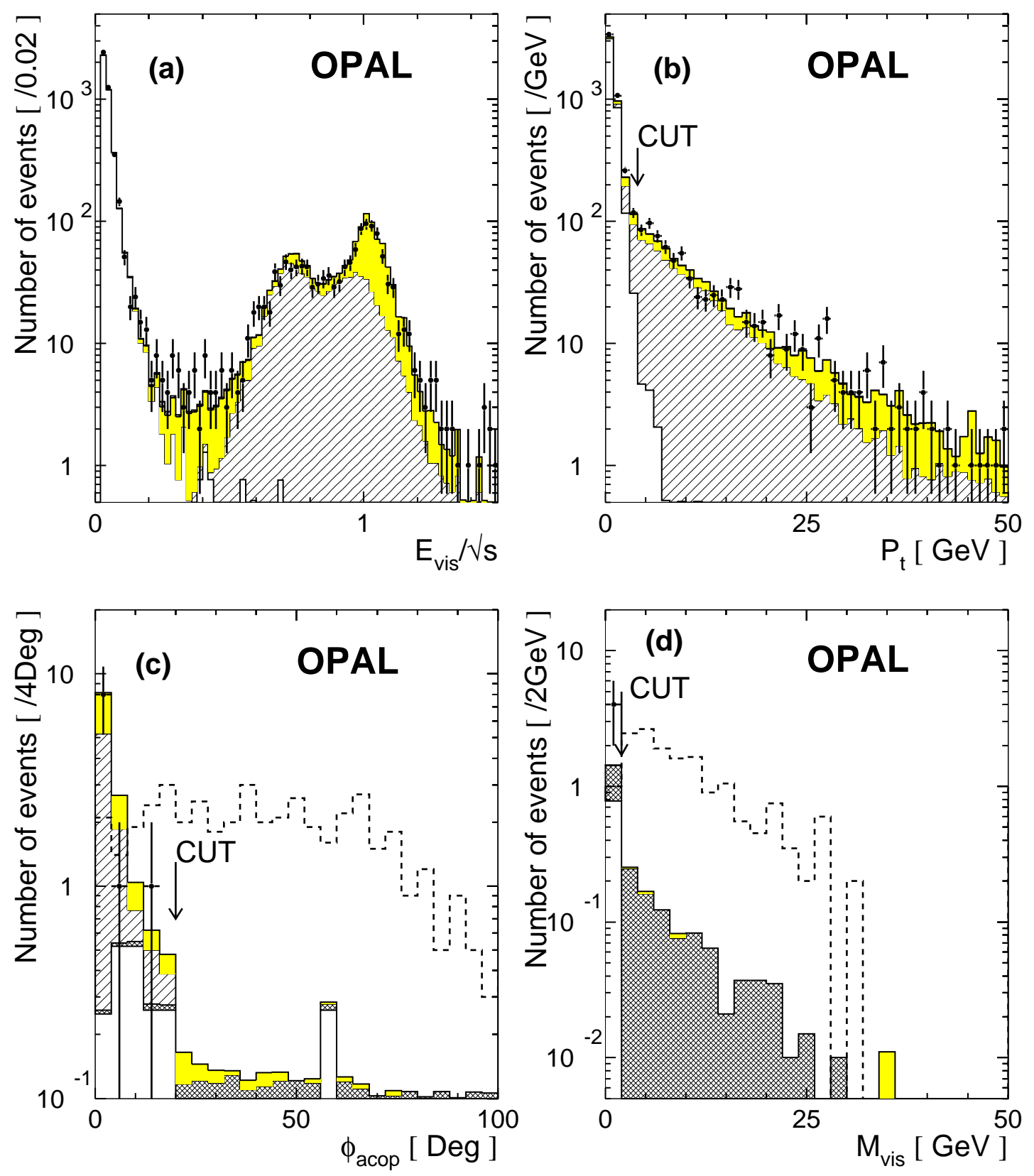

Figure 1: (a) The distribution of the total visible energy normalised to $\sqrt{s}$ and (b) the distribution of $P_{t}$ after cut (1). (c) The acoplanarity angle distribution after cut (A1). The prediction for $\tilde{\chi}_{1}^{+} \tilde{\chi}_{1}^{-}$events is also shown for $m_{\tilde{\chi}_{1}^{+}}=60 \mathrm{GeV}$ and $m_{\tilde{\chi}_{1}^{0}}=30 \mathrm{GeV}$ with a production cross section of $20 \mathrm{pb}$ as a dashed histogram. (d) The visible mass distribution for monojet events. The prediction for $\tilde{\chi}_{1}^{0} \tilde{\chi}_{2}^{0}$ events is also shown for $m_{\tilde{\chi}_{2}^{0}}=80 \mathrm{GeV}$ and $m_{\tilde{\chi}_{1}^{0}}=30 \mathrm{GeV}$ with a production cross section of $5 \mathrm{pb}$ as a dashed histogram. In all the plots, the hatched area indicates the prediction for $q \bar{q}(\gamma)$ events, the grey area for $\ell^{+} \ell^{-}(\gamma)$ events, the open area for two photon processes, and the double hatched area for four fermion processes $\left(\nu \bar{\nu} \ell^{+} \ell^{-}, \nu \bar{\nu} \mathrm{q} \overline{\mathrm{q}}\right.$, $\nu \ell \mathrm{q} \overline{\mathrm{q}}^{\prime}, \tau^{+} \tau^{-} \mathrm{q} \overline{\mathrm{q}}, \tau^{+} \tau^{-} \tau^{+} \tau^{-}$and $\left.\tau^{+} \tau^{-} \mu^{+} \mu^{-}\right)$. 

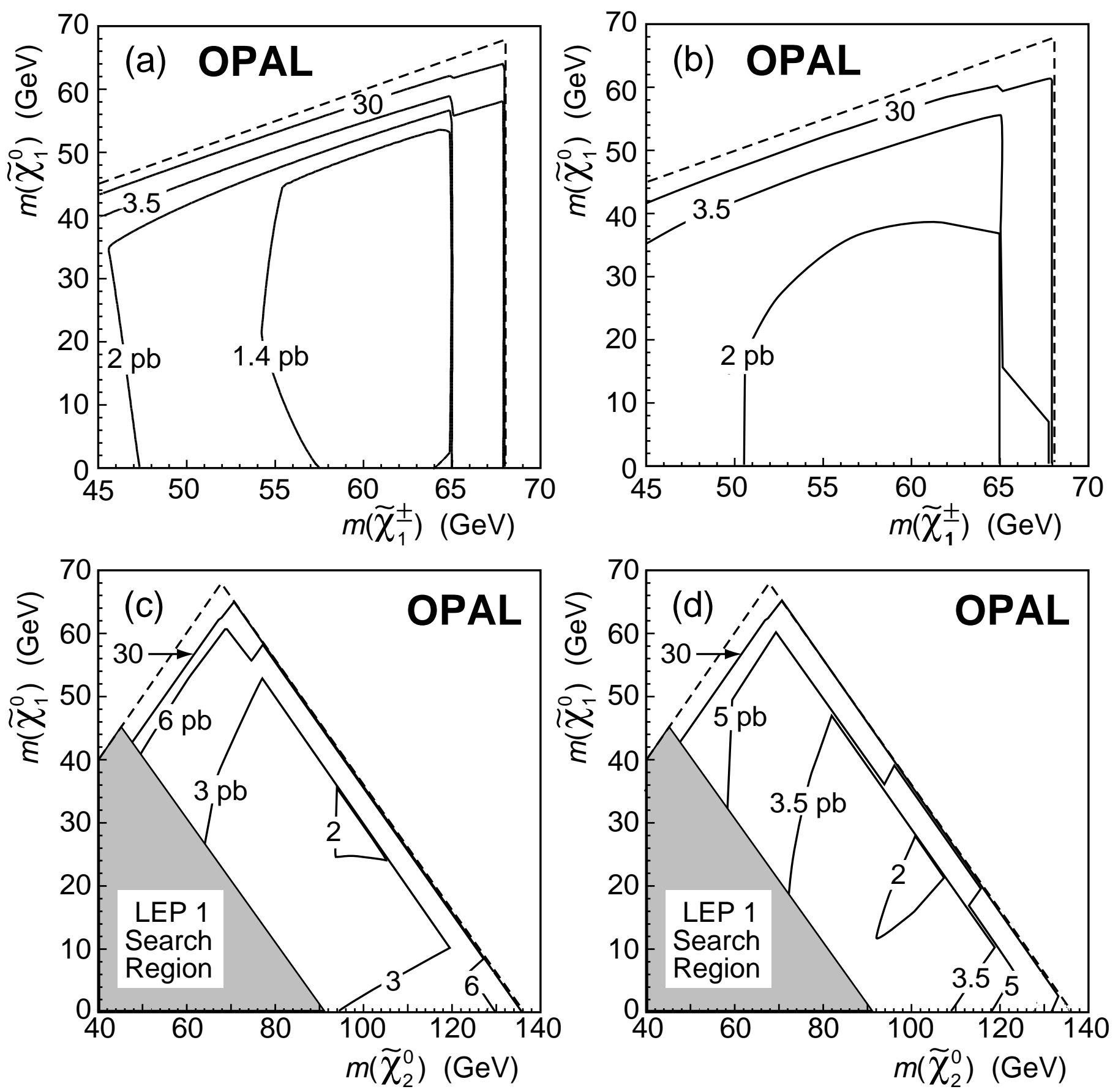

Figure 2: The 95\% C.L. upper limits for the production cross sections at $\sqrt{s}=136 \mathrm{GeV}$ for $\tilde{\chi}_{1}^{+} \tilde{\chi}_{1}^{-}$assuming (a) $\tilde{\chi}_{1}^{+} \rightarrow \tilde{\chi}_{1}^{0} \mathrm{~W}^{*+}$ with $100 \%$ branching fraction or (b) $\chi_{1} \rightarrow \tilde{\chi}_{1}^{0} \ell^{+} \nu$ via $\tilde{\ell}$ or $\tilde{\nu}$ with $100 \%$ branching fraction. The contours of the upper limits for the $\tilde{\chi}_{1}^{0} \tilde{\chi}_{2}^{0}$ cross sections at $\sqrt{s}=136 \mathrm{GeV}$ are shown for $(\mathrm{c}) \tilde{\chi}_{2}^{0} \rightarrow \tilde{\chi}_{1}^{0} \mathrm{Z}^{*}$ with $100 \%$ branching fraction, or (d) $\tilde{\chi}_{2}^{0} \rightarrow \tilde{\chi}_{1}^{0} \ell^{+} \ell^{-}$ via $\tilde{\ell}$ with $100 \%$ decay branching fraction. The regions $\left(m_{\tilde{\chi}_{1}^{0}}+m_{\tilde{\chi}_{2}^{0}}\right)<M_{\mathrm{Z}}$, which are accessible at LEP 1 , are not considered in this analysis and are indicated in the plots. The kinematical boundaries for production and decay at $\sqrt{s}=136 \mathrm{GeV}$ are shown by dashed lines. 

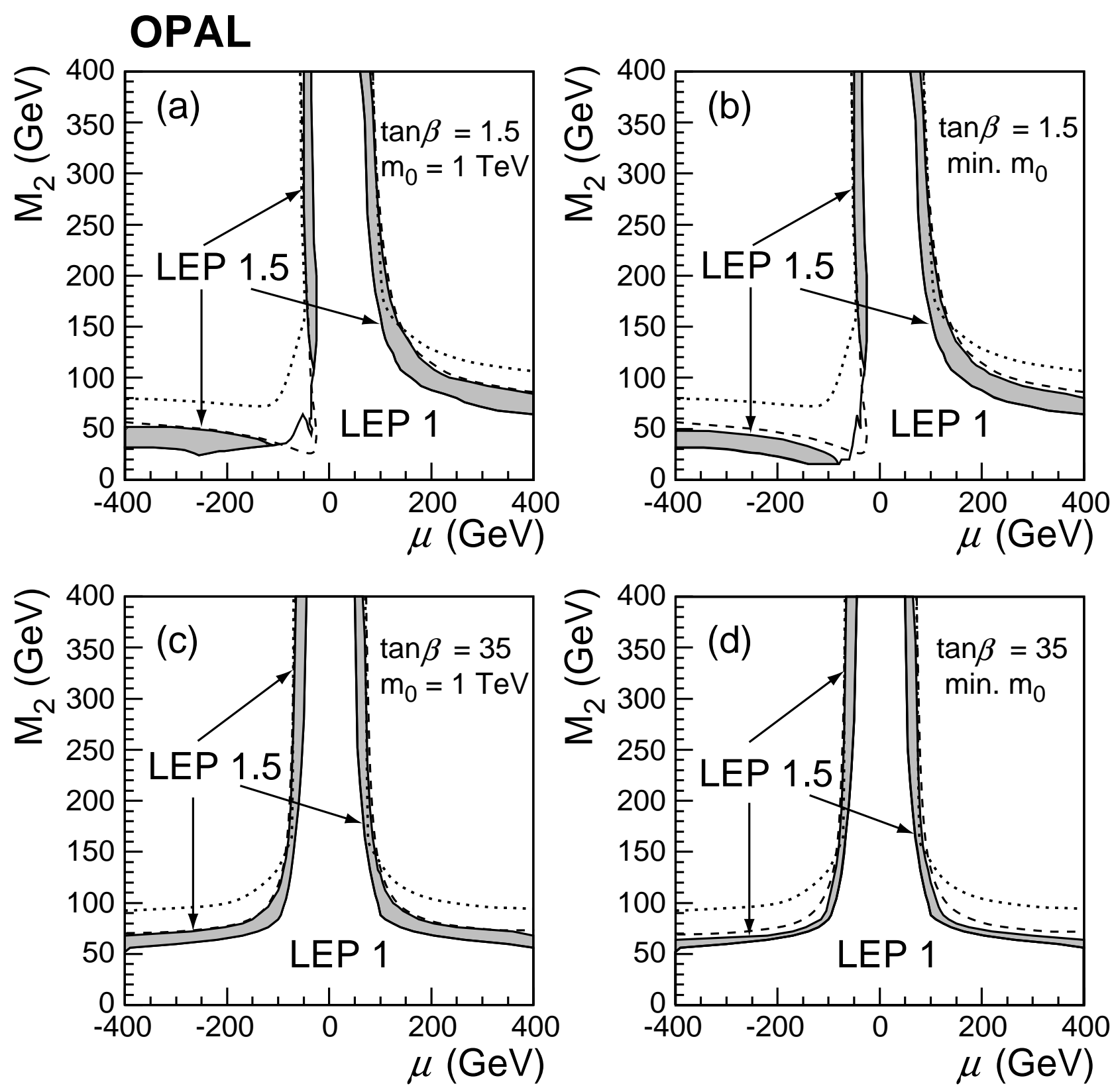

Figure 3: The exclusion contours in the $M_{2}-\mu$ plane for the case (a) $m_{0}=1 \mathrm{TeV}$ and (b) minimum $m_{0}$ with $\tan \beta=1.5$. The same plots are shown for $\tan \beta=35$ (c and $\mathrm{d}$ ). The minimum $m_{0}$ is defined to be the smallest $m_{0}$ possible to comply with the limits on $\tilde{\ell}$ and $\tilde{\nu}$ at LEP 1 . The region excluded by the analysis of LEP 1 data is also shown. The kinematical boundary at $\sqrt{s}=136 \mathrm{GeV}$ for $\tilde{\chi}_{1}^{+} \tilde{\chi}_{1}^{-}$production is shown by dashed lines and for $\tilde{\chi}_{1}^{0} \tilde{\chi}_{2}^{0}$ production as dotted lines. 
OPAL
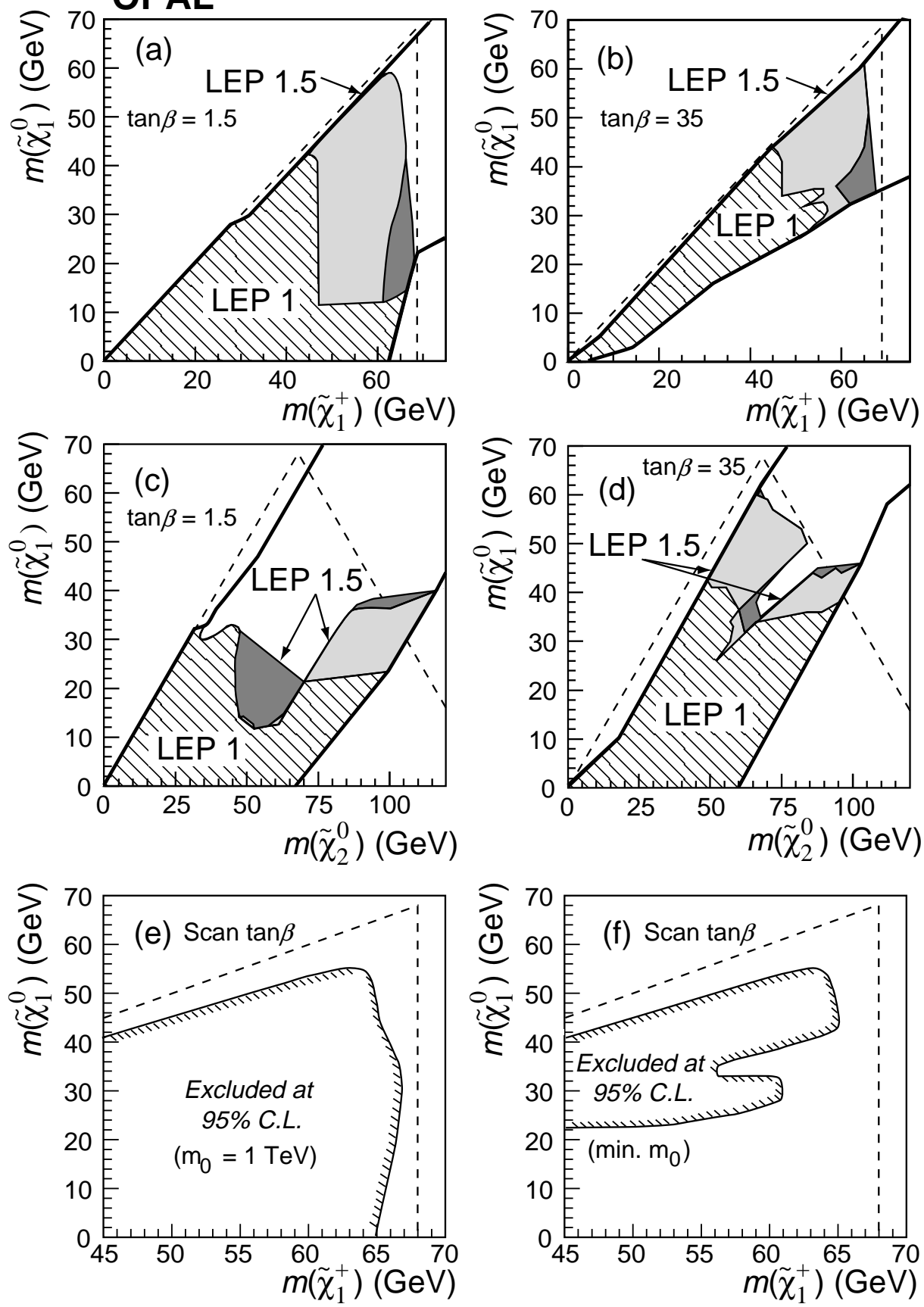

Figure 4: The 95\% C.L. excluded region in the $m_{\tilde{\chi}_{1}^{0}}-m_{\tilde{\chi}_{1}^{+}}$plane within the framework of the MSSM for the case of minimum $m_{0}$ (light shaded region) and $m_{0}=1 \mathrm{TeV}$ (extending to dark shaded region) for (a) $\tan \beta=1.5$ and (b) $\tan \beta=35$. The region excluded by the analysis of LEP 1 data is also shown. The thick solid lines bound the region that is physically accessible for the given region of MSSM parameter space. The kinematical boundaries for production and decay at $\sqrt{s}=136 \mathrm{GeV}$ are shown by dashed lines. Similar plots for neutralino masses for (c) $\tan \beta=1.5$ and (d) $\tan \beta=35$. The regions excluded at $95 \%$ C.L. (e-f) in the $m_{\tilde{\chi}_{1}^{0}-m_{\tilde{\chi}_{1}^{+}} \text {plane }}$ conservatively obtained by scanning through $\tan \beta(\tan \beta>1)$ to find the smallest number of events expected (sum of $\tilde{\chi}_{1}^{+} \tilde{\chi}_{1}^{-}, \tilde{\chi}_{1}^{0} \tilde{\chi}_{2}^{0}$ and $\tilde{\chi}_{2}^{0} \tilde{\chi}_{2}^{0}$ events after all the cuts) at any point of the plane and value of $\tan \beta$ for (e) $m_{0}=1 \mathrm{TeV}$ and (f) minimum $m_{0}$. 